\title{
Nondestructive Evaluation of Functionally Graded Subsurface Damage on Cylinders in Nuclear Installations Based on Circumferential SH Waves
}

\author{
Zhen Qu, ${ }^{1,2}$ Xiaoqin Shen, ${ }^{2}$ and Xiaoshan $\mathrm{Cao}^{1,3}$ \\ ${ }^{1}$ School of Civil Engineering and Architecture, Xian University of Technology, Xian 710048, China \\ ${ }^{2}$ School of Sciences, Xian University of Technology, Xian 710054, China \\ ${ }^{3}$ State Key Laboratory of Transducer Technology, Chinese Academy of Sciences, Shanghai 200050, China
}

Correspondence should be addressed to Xiaoqin Shen; xqshen@xaut.edu.cn

Received 30 June 2016; Accepted 7 September 2016

Academic Editor: Xing Chen

Copyright (C) 2016 Zhen Qu et al. This is an open access article distributed under the Creative Commons Attribution License, which permits unrestricted use, distribution, and reproduction in any medium, provided the original work is properly cited.

\begin{abstract}
Subsurface damage could affect the service life of structures. In nuclear engineering, nondestructive evaluation and detection of the evaluation of the subsurface damage region are of great importance to ensure the safety of nuclear installations. In this paper, we propose the use of circumferential horizontal shear $(\mathrm{SH})$ waves to detect mechanical properties of subsurface regions of damage on cylindrical structures. The regions of surface damage are considered to be functionally graded material (FGM) and the cylinder is considered to be a layered structure. The Bessel functions and the power series technique are employed to solve the governing equations. By analyzing the SH waves in the 12Cr-ODS ferritic steel cylinder, which is frequently applied in the nuclear installations, we discuss the relationship between the phase velocities of $\mathrm{SH}$ waves in the cylinder with subsurface layers of damage and the mechanical properties of the subsurface damaged regions. The results show that the subsurface damage could lead to decrease of the SH waves' phase velocity. The gradient parameters, which represent the degree of subsurface damage, can be evaluated by the variation of the SH waves' phase velocity. Research results of this study can provide theoretical guidance in nondestructive evaluation for use in the analysis of the reliability and durability of nuclear installations.
\end{abstract}

\section{Introduction}

In many engineering fields, such as the nuclear and aerospace industries, nondestructive evaluation of damage is important for enhancing the lifetime and ensuring the reliability of devices, and this is especially pertinent for nuclear equipment. Generally speaking, the corrosive environments, fatigue under periodic mechanical or thermal loading during operations, and nuclear radiation exposure might lead to the material's subsurface damage [1-6]. Acoustic waves are typically good tools for detecting the properties of subsurface damaged regions in structures [7]. The influence of subsurface damage on surface waves should also be investigated.

Paehler et al. [8] found that the elastic coefficient in the subsurface regions of silicon wafers with subsurface damage is a variable dependent upon depth based on the Rayleigh waves dispersion relation obtained experimentally. However, in that experiment, the subsurface region was corroded step by step. Therefore, despite the use of acoustic waves by the researchers, that technique is not a truly nondestructive evaluation. Cao et al. [9] proposed that regions of subsurface damage should be treated as functionally graded material (FGM) layers of the material. Then guide waves in the FGM model could be applied to detect subsurface damage in structures.

Some researchers have attempted to simplify the problem by theoretically assuming that FGM material with multilayered structures has homogeneous but discontinuous properties. Daros [10] used the boundary element method to explain SH wave propagation in inhomogeneous media. Potel et al. [11] described SH waves along two isotropic solid plates with integral formulation.

In other articles, many researchers use the special functions method or transfer matrix method to describe the 


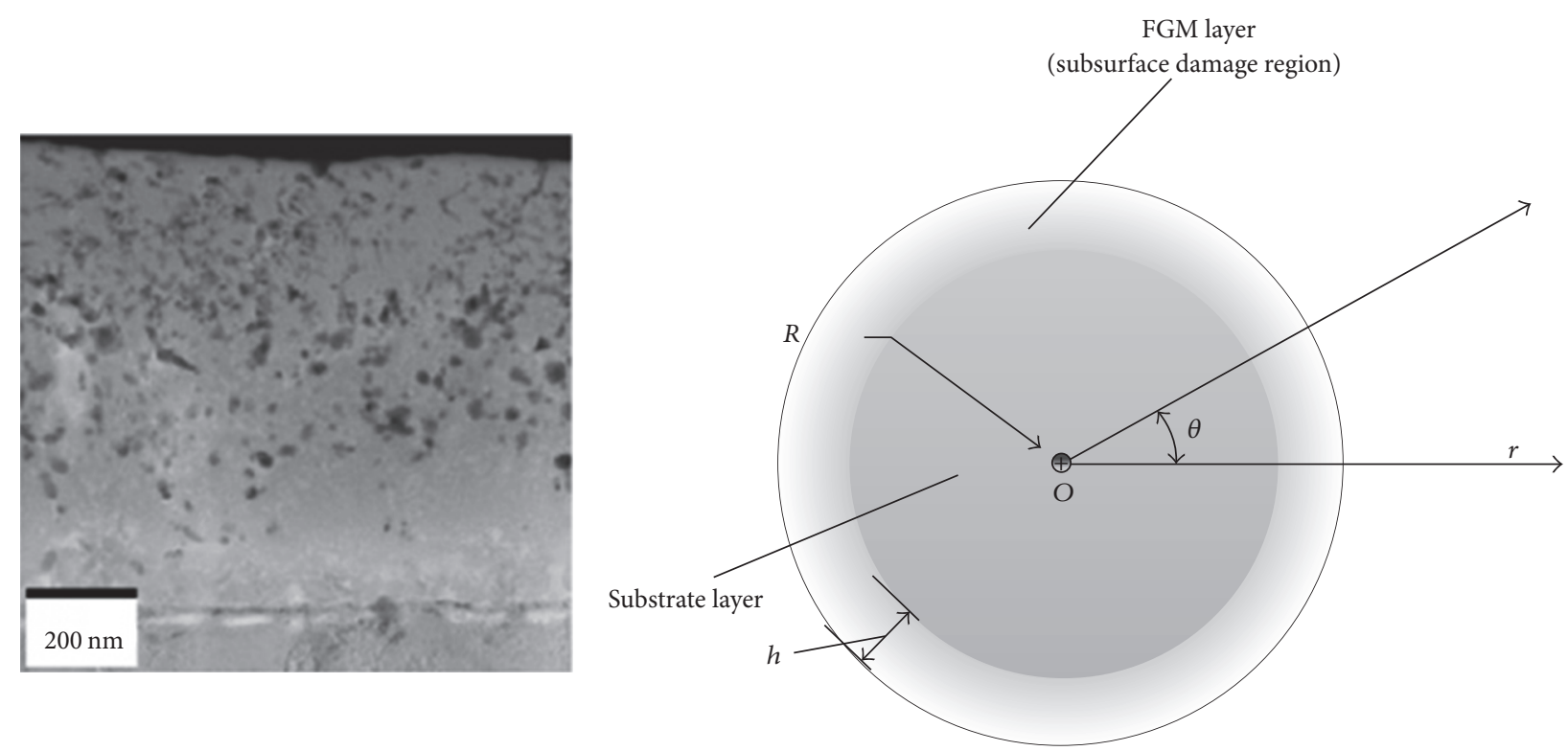

FIGURE 1: Structure of the cylinder with surface damage (see [23]).

problem of $\mathrm{SH}$ wave propagation in the layered structure [12-15]. Some reports have been published on the analysis of the propagation properties of Love waves in FGM layered structures with the Wentzel-Kramers-Brillouin (WKB) method [16-18]. The analytic solutions with the special functions method to describe the Love wave, B-G wave, and horizontal shear waves in FGM structures have been reported by other researchers. In these reports, the WKB and special functions methods can only be used at high frequencies and are only good in certain cases. The power series technique has been used by Cao et al. [19] to resolve the difficulty of the variable coefficient differential equation and fit for general cases. There is a substantial body of research concerning wave propagation in FGM or layered structures focusing on half-space or layered films, but studies which focused on cylindrical structures are less common. Zhu et al. [20] used the reverberation-ray matrix formulation for cylindrical coordinates to discuss the ways in which material inhomogeneity primarily affects wave propagation for high modes. Legendre polynomial series method was used by $\mathrm{Yu}$ et al. [21] to solve coupled wave equations with variable coefficients.

Most studies on subsurface damage are focused on flat structures, but the curvature of the structure also influences wave propagation [22]. In our study, we consider the propagation of the circumferential $\mathrm{SH}$ wave in cylindrical structures with surface damage and analyze the surface damage layer as an FGM layer. In this layer, the material properties are a function of the cylinder thickness. In the FGM layer, the power series technique is used to solve the governing equations as a variable coefficient differential with cylindrical coordinates. In the rest of the cylinder, the special functions method is applied. Based on a numerical example, we discuss the dispersion relation of the circumferential $\mathrm{SH}$ wave and the influence of gradient parameters on $\mathrm{SH}$ wave propagation.

\section{Statement of the Problem}

As shown in Figure 1, the surface damage layer is an FGM layer with thickness $h$, while $R$ is the thickness of substrate layer. This is based upon the assumption that $\mathrm{SH}$ surface waves propagate along the circumferential direction, and the surface stress is free. Considering that the thickness of the substrate is greater than that of the FGM layer, it is assumed that the substrate is a half-space. According to generalized Hooke's law, the constitutive equations can be expressed as follows:

$$
\sigma_{i j}=c_{i j k l} \varepsilon_{k l}
$$

where $\sigma_{i j}$ and $\varepsilon_{k l}$ are the stress and strain tensors and $c_{i j k l}$ is the elastic coefficient. In the FGM layer, $c_{i j k l}$ are a function of layer thickness, and in the substrate $c_{i j k l}$ are constants.

The motion equation is then given as follows:

$$
\sigma_{i j, j}=\rho \ddot{u}_{i}
$$

where $\rho$ is the material density, the subscript $i$ denotes the $i$ th direction in the corresponding coordinates, $u_{i}$ is the displacement of $i$ th direction, the dot $(\cdot)$ denotes the differential of time, and $j$ preceded by a comma means that there is space differentiation of the relevant coordinate $x_{j}$. In this paper, we choose the cylindrical coordinates, so $i$ or $j$ can be denoted by $r, \theta$, and $z$. follows:

The displacement and the strain tensors are expressed as

$$
\varepsilon_{i j}=\frac{1}{2}\left(u_{i, j}+u_{j, i}\right)
$$

For the SH surface waves propagating along the circumferential direction, let the displacement component be described 
as $u_{r}=u, u_{\theta}=v$, and $u_{z}=w$, and $u, v$, and $w$ can be expressed as

$$
\begin{aligned}
& u=v=0, \\
& w=w(r, \theta, t) .
\end{aligned}
$$

Equation (1) can be simplified as

$$
\begin{gathered}
\tau_{r z}=c_{44} \varepsilon_{r z}, \\
\tau_{\theta z}=c_{44} \varepsilon_{\theta z} .
\end{gathered}
$$

Using (2), (3), (4), and (5), we can induce the governing equation of displacement for corresponding material layers.

In the substrate layer, $w^{(1)}$ is defined as the mechanical displacement component. The governing equation for $\mathrm{SH}$ waves in the substrate layer $r \in(0, R]$ can be obtained:

$$
c_{440} \frac{\partial^{2} w^{(1)}}{\partial r^{2}}+c_{440} \frac{1}{r} \frac{\partial w^{(1)}}{\partial r}+\frac{1}{r^{2}} \frac{\partial^{2} w^{(1)}}{\partial \theta^{2}}=\rho \frac{\partial^{2} w^{(1)}}{\partial t^{2}},
$$

where $c_{440}$ is the constant material elastic coefficient and $\rho$ is the material density of the substrate layer.

In the FGM layer, we can define the mechanical displacement component as $w^{(2)}$. Therefore, the governing equation for $\mathrm{SH}$ waves in the FGM layer can be described as $r \in$ $[R, R+h]$. Consider

$$
\begin{gathered}
c_{44}(r) \frac{\partial^{2} w^{(2)}}{\partial r^{2}}+c_{44}^{\prime}(r) \frac{\partial w^{(2)}}{\partial r}+c_{44}(r) \frac{1}{r} \frac{\partial w^{(2)}}{\partial r} \\
+\frac{1}{r^{2}} \frac{\partial^{2} w^{(2)}}{\partial \theta^{2}}=\rho(r) \frac{\partial^{2} w^{(2)}}{\partial t^{2}} .
\end{gathered}
$$

Because the material parameters are functions of layer thickness in the FGM layer, $c_{44}(r)$ is used to denote the function of elastic coefficients, $\rho(r)$ is the function of material density, and $c_{44}^{\prime}(r)$ is the differentiation with coordinate $r$.

Additionally, for the problem of $\mathrm{SH}$ waves propagating in the cylindrical structure with subsurface damage, the following boundary conditions and continuity conditions should be satisfied:

(1) Traction-free boundary conditions at $r=R+h$ :

$$
\tau_{r z}^{(2)}(R+h, \theta)=0
$$

(2) The continuity conditions at $r=R$ :

$$
\begin{aligned}
& w^{(1)}(R, \theta, t)=w^{(2)}(R, \theta, t), \\
& \tau_{r z}^{(1)}(R, \theta, t)=\tau_{r z}^{(2)}(R, \theta, t) .
\end{aligned}
$$

The superscripts "(1) and (2)" in the above-mentioned boundary conditions and continuity conditions denote the mechanical quantities in the substrate layer and the FGM layer, respectively.

\section{Solution to the Problem}

For the mentioned $\mathrm{SH}$ waves in the substrate layer of the cylinder, the solution to the governing equations can be assumed in the following form:

$$
w^{(1)}(r, \theta, t)=W^{(1)}(r) \exp [i(k h \theta+k R \theta-\omega t)],
$$

where $i=\sqrt{-1}, \omega=k c$ is the circular frequency, and $k$, $c$, and $W^{(1)}(r)$ are the wave number, the phase velocity, and the unknown amplitudes function of the displacement, respectively.

Substituting (10) into (6), we obtain Bessel's equation of order $l$ as follows:

$$
\frac{\mathrm{d}^{2} W^{(1)}}{\mathrm{d} r^{2}}+\frac{1}{r} \frac{\mathrm{d} W^{(1)}}{\mathrm{d} r}+\left(u-\frac{l^{2}}{r^{2}}\right) W^{(1)}=0,
$$

where $u=\rho^{(1)} \omega^{2} / c_{440}$ and $l=k R+k h$. By using the special functions method, the solution of the equation can be expressed as follows:

$$
W^{(1)}=C_{1} J_{l}(u r)+C_{2} Y_{l}(u r)
$$

where $C_{1}$ and $C_{2}$ are the undetermined constants and $J_{l}(x)$ and $Y_{l}(x)$ are first-class and second-class Bessel's functions with order $l$. When $r \rightarrow 0, W^{(1)}(r)$ must have a finite value, so $C_{2}=0$ can be deduced. Therefore, (12) can be simplified as

$$
W^{(1)}=C_{1} J_{l}(u r)
$$

Similarly, we can arrive at the solution of the governing equations in the FGM layer with the following formula:

$$
w^{(2)}(r, \theta, t)=W^{(2)}(r) \exp [i(k R \theta+k h \theta-\omega t)],
$$

where $W^{(2)}(r)$ is the unknown amplitudes function of the displacement. Substituting (14) into (7), we obtain

$$
\begin{gathered}
c_{44}(r) \frac{\mathrm{d}^{2} W}{\mathrm{~d} r^{2}}+c_{44}^{\prime}(r) \frac{\mathrm{d} W}{\mathrm{~d} r}+\frac{1}{r} c_{44}(r) \frac{\mathrm{d} W}{\mathrm{~d} r} \\
-\frac{l^{2}}{r^{2}} c_{44}(r) W+\rho \omega^{2} W=0 .
\end{gathered}
$$

By letting $\widehat{r}=(r-R) / h$, (15) can be rewritten as an ordinary differential equation with respect to $\widehat{r}$ :

$$
\begin{aligned}
(\widehat{r}+ & \left.\frac{R}{h}\right)^{2} c_{44}(\widehat{r}) \frac{\mathrm{d}^{2} W}{\mathrm{~d} \widehat{r}^{2}}+\left(\widehat{r}+\frac{R}{h}\right)^{2} \frac{\mathrm{d} c_{44}(\widehat{r})}{\mathrm{d} \widehat{r}} \\
& +\left(\widehat{r}+\frac{R}{h}\right) c_{44}(\widehat{r}) \frac{\mathrm{d} W}{\mathrm{~d} \widehat{r}}-l^{2} c_{44}\left(\widehat{r}^{2}\right) W \\
& +(h \widehat{r}+R)^{2} \rho\left(\widehat{r}^{2}\right) \omega^{2} W=0 .
\end{aligned}
$$

To solve this ordinary differential equation with the variable coefficient, we assume that the material parameters 
functions can be expanded as the power series with respect to $\widehat{r}^{n}$ by using the power series technique as follows:

$$
\begin{gathered}
c_{44}(\widehat{r})=\sum_{n=0}^{\infty} m_{n}^{1} \widehat{r}^{n}, \\
\rho(\widehat{r})=\sum_{n=0}^{\infty} m_{n}^{2} \widehat{r}^{n} .
\end{gathered}
$$

Hence, with the material parameters subjected to the power series technique, the amplitude function should have the same form as

$$
W^{(2)}(\widehat{r})=\sum_{n=0}^{\infty} a_{n} \widehat{r}^{n}
$$

where $m_{n}^{k}=(1 / n !)\left(\mathrm{d}^{n} f_{k} / \mathrm{d} r^{n}\right), k=1,2$, and $a_{n}$ are the coefficients of the power series. $f_{k}$ is the function which describes the variation of material parameters with layer thickness, and $m_{n}^{k}$ can be determined by the relationships between the function and its Taylor expansion. Substituting (17) and (18) into (16), we get the following equation:

$$
\begin{aligned}
\left(\widehat{r}+\frac{R}{h}\right)^{2}\left(\sum_{n=0}^{\infty} m_{n}^{1} \widehat{r}^{n}\right)\left(\sum_{n=0}^{\infty} n(n-1) a_{n} \widehat{r}^{n-2}\right) \\
+\left(\widehat{r}+\frac{R}{h}\right)^{2}\left(\sum_{n=0}^{\infty} n m_{n}^{1} \widehat{r}^{n-1}\right)\left(\sum_{n=0}^{\infty} n a_{n} \widehat{r}^{n-1}\right) \\
+\left(\widehat{r}+\frac{R}{h}\right)\left(\sum_{n=0}^{\infty} m_{n}^{1} \widehat{r}^{n}\right)\left(\sum_{n=0}^{\infty} n a_{n} \widehat{r}^{n-1}\right) \\
+l^{2}\left(\sum_{n=0}^{\infty} m_{n}^{1} \widehat{r}^{n}\right)\left(\sum_{n=0}^{\infty} a_{n} \widehat{r}^{n}\right) \\
+(h \widehat{r}+R)^{2} \omega^{2}\left(\sum_{n=0}^{\infty} m_{n}^{2} \widehat{r}^{n}\right)\left(\sum_{n=0}^{\infty} a_{n} \widehat{r}^{n}\right)=0 ;
\end{aligned}
$$

the coefficients of $\hat{r}^{n}$ on the two sides of (19) must equal each other. Therefore, the relation of $a_{n}$ can be obtained:

$$
\begin{aligned}
& \sum_{j=0}^{n}\left[n(n-j)-l^{2}\right] m_{j}^{1} a_{n-j}+\left(\frac{R}{h}\right) \\
& \cdot \sum_{j=0}^{n}[(n-j+1)(2 n+1)] m_{j}^{1} a_{n-j+1}+\left(\frac{R}{h}\right)^{2} \\
& \cdot \sum_{j=0}^{n}[(n-j+2)(n+1)] m_{j}^{1} a_{n-j+2} \\
& +\sum_{j=0}^{n}\left(h^{2} \omega^{2} m_{j}^{2} a_{n-j-2}+2 h R \omega^{2} m_{j}^{2} a_{n-j-1}\right. \\
& \left.+R^{2} \omega^{2} m_{j}^{2} a_{n-j}\right)=0 .
\end{aligned}
$$

Let $a_{-2}=a_{-1}=0$; when $n \geq 2$, (15) is a recurrence equation of $a_{n}$ and can be solved through a specific value of $a_{0}, a_{1}$. Then the amplitudes function is redescribed as

$$
W^{(2)}(\widehat{r})=\sum_{j=2}^{3} C_{j}\left(\sum_{n=0}^{\infty} a_{n j} \widehat{r}^{n}\right),
$$

where $\left(a_{0 j}, a_{1 j}\right)=I$ and $I$ is a $2 \times 2$ unit matrix. Then, substituting (13) and (21) into the boundary and continuity conditions, the linear equation with $C_{i}, i=1,2,3$, can be obtained, and this equation must have a nontrivial solution. From the sufficient and necessary condition of homogeneous linear equation, we know that the determinant of the coefficient matrix is equal to zero, which expresses the dispersion relation for $\mathrm{SH}$ waves.

$$
\left|T_{i j}\right|=0,
$$

where $T_{i j}, i, j=1,2,3$, can be written as follows:

$$
\begin{aligned}
& T_{11}=0, \\
& T_{1 j}=\frac{1}{h} c_{44}(R+h)\left(\sum_{n=0}^{\infty} n a_{n j}\right), \quad j=2,3, \\
& T_{21}=J_{l}(u R), \\
& T_{22}=-a_{02}, \\
& T_{23}=-a_{03}, \\
& T_{31}=c_{44}^{(1)} J_{l}^{\prime}(u R), \quad \\
& T_{3 j}=-c_{44}(R) a_{1 j}, \quad j=2,3 .
\end{aligned}
$$

\section{Numerical Examples}

Next, the numerical examples are given to study the propagation behavior of $\mathrm{SH}$ waves in cylindrical structures and to graphically show the dispersion relation. Suppose that the cylinder with surface damage is a composite of an FGM layer and a homogeneous substrate with the same material. This is a simplified model of the nuclear detector equipment used in nuclear engineering. In the substrate layer, the material parameters $c_{440}$ and $\rho$ are constant, and in the FGM layer these parameters become functions with the layer thickness. Therefore, in order to describe the surface damage, we describe the two types of the gradient functions as follows:

Type A:

$$
f(\widehat{r})=\exp (-p \widehat{r})
$$

Type B:

$$
f(\widehat{r})=1-p \widehat{r},
$$

where $p$ is the gradient parameter. Then the parameter functions with thickness can be denoted as

$$
c_{44}(\widehat{r})=c_{440} f(\widehat{r}) .
$$




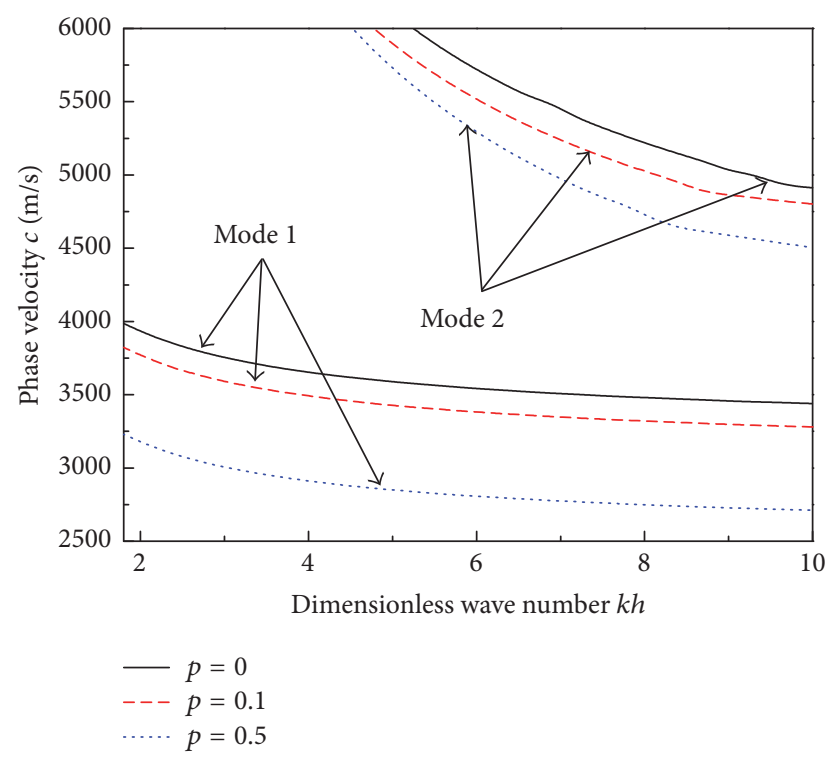

FIGURE 2: Comparing the dispersion relation of the $\mathrm{SH}$ waves with the different gradient parameter $p$ for Type A.

Within the equipment of nuclear applications, 12CrODS has great applicability because of its good mechanical properties under irradiation and high temperatures [1]. In this paper, $12 \mathrm{Cr}$-ODS ferritic steel is selected as the cylinder's material and its material parameters are given as $c_{440}=$ $80 \mathrm{GPa}$ and $\rho=7.85 \times 10^{3} \mathrm{~kg} / \mathrm{m}^{3}$. The thickness of the FGM layer and substrate is $h=0.003 \mathrm{~m}$, and the radius of the cylinder is $R=1 \mathrm{~m}$. Generally, subsurface damage has great influence on the material's mechanic parameters, so we assume that $c_{44}(\widehat{r})$ is a function with the layer thickness and the density $\rho$ is constant in the FGM layer. Next the dispersion relation of the $\mathrm{SH}$ waves in the cylindrical structure with surface damage is discussed.

Figure 2 shows the dispersion curves of the $\mathrm{SH}$ wave that propagates in the cylinder with different gradient parameter $p ; p=0$ indicates that the material is homogeneous without surface damage and describes the different modes of the wave propagation when the mechanical parameters function as Type A in the surface damage layer. Obviously, with the increase in the gradient parameter $p$, the phase velocity of the wave is decreased. Figure 3 shows how the mechanical parameters function as Type B influencing the surface damage. The change tendency of the phase velocity is similar to that seen in Figure 2. For both of these types of surface damage, the gradient parameter influences the dispersion relation of the $\mathrm{SH}$ wave to a greater extent in mode 2. For every gradient parameter, with the increase of wave number $k$, the phase velocity is decreased.

We also have investigated the influence of the gradient parameter on the phase velocity. For given $k h$, such as $k h=$ $2 \pi$, the relationship between the gradient parameter $p$ and the absolute value of the increment of phase velocity $|\Delta c|$ has been discussed, where $|\Delta c|=\bar{c}-c, \bar{c}$ is the phase velocity in cylinder without subsurface damage, and $c$ is the phase velocity in

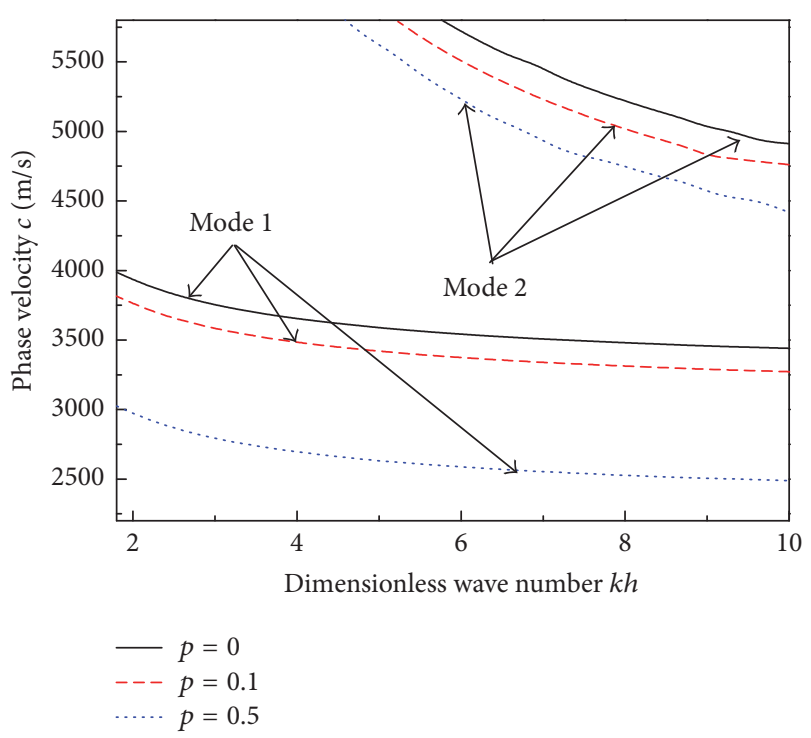

FIgure 3: Comparing the dispersion relation of the $\mathrm{SH}$ waves with the different gradient parameter $p$ for Type B.

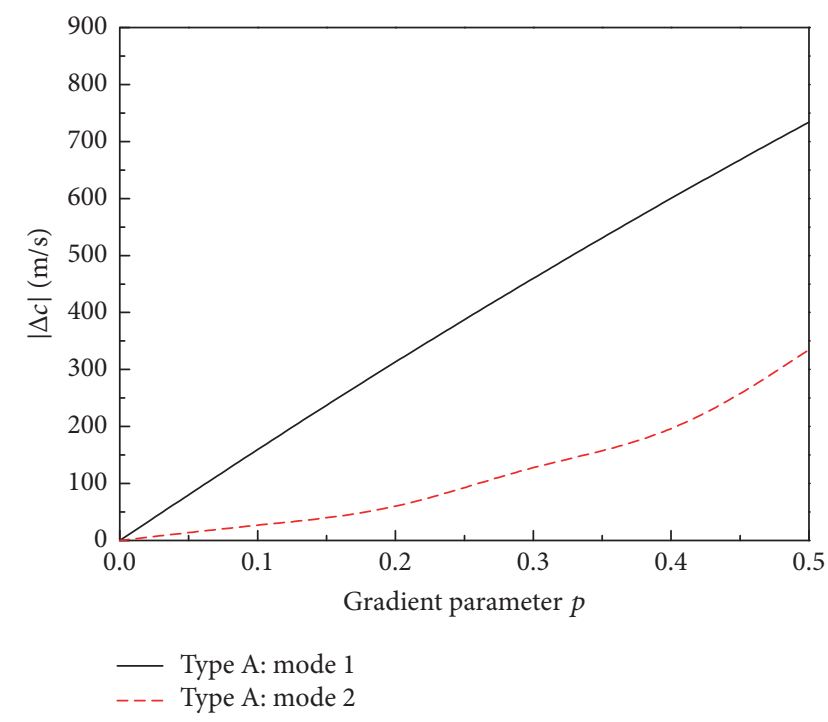

FIgURE 4: The influence of gradient parameter on the increment of phase velocity with subsurface damage Type A.

different gradient parameter. As shown in Figures 4 and 5, with the increase of gradient parameter $p,|\Delta c|$ is increased for both subsurface damage Type A and subsurface damage Type $B$. It means that the larger gradient parameter could cause the significant change tendency of phase velocity of $\mathrm{SH}$ wave. In Type $\mathrm{A}$, the changing in the phase velocity is more stable and notable in mode 1 than in mode 2 . In Type B, although the changing tendency of the phase velocity holds steady in mode 1 , the increasing of $|\Delta c|$ is more obvious in mode 2. These results indicate that it is possible to detect subsurface damage on cylindrical structure through the change of the phase velocity in specific circumstances. Therefore, these properties of SH waves can be applied for nondestructive evaluation of subsurface damage on nuclear installations. 


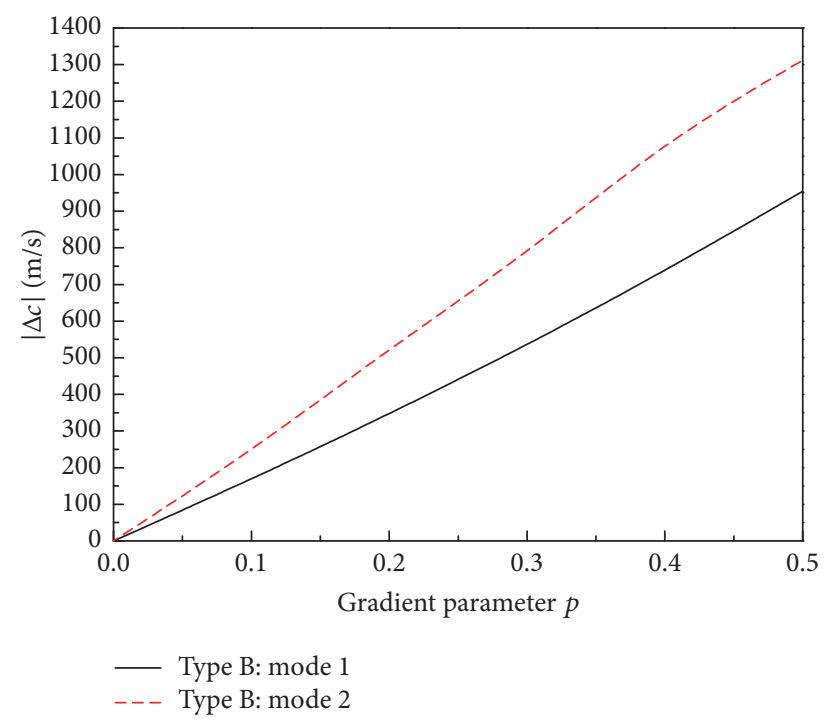

FIGURE 5: The influence of gradient parameter on the increment of phase velocity with subsurface damage Type B.

\section{Conclusions}

Using the series technique, the circumferential SH waves in cylinders with surface damage were analyzed. The results showed that the gradient parameters affect the propagation of the waves, and the rate of change of the phase velocity is more stable in mode 1 than in mode 2 . With the change in the subsurface damage type, the propagation of $\mathrm{SH}$ waves is significantly different. We found that it is possible to check the equipment for surface damage using the change value of the phase velocity of SH waves, and this provides us with a method for identifying the type of damage we encountered. These results could also be used as theoretical guidance in the nondestructive evaluation of the reliability and durability of nuclear installations.

\section{Competing Interests}

The authors declare that they have no competing interests.

\section{Acknowledgments}

The authors gratefully acknowledge the support by the National Natural Science Foundation of China (nos. 11572244, 11571275, and U1630144), the Natural Science Foundation of Shaanxi Province (nos. 2015JQ1001 and 2015GY021), and the Open State Key Laboratories of Transducer Technology (no. SKT1506).

\section{References}

[1] Z. B. Yang, B. F. Hu, H. Kinoshita, H. Takahashi, and S. Watanabe, "Effect of hydrogen ion/electron dual-beam irradiation on micro structural damage of a 12Cr-ODS ferrite steel," Journal of Nuclear Materials, vol. 398, no. 1-3, pp. 81-86, 2010.
[2] M. A. Rana, "Swelling and structure of radiation induced nearsurface damage in CR-39 and its chemical etching," Radiation Measurements, vol. 47, no. 1, pp. 50-56, 2012.

[3] H. Sako, H. Matsuhata, M. Sasaki et al., "Micro-structural analysis of local damage introduced in subsurface regions of $4 \mathrm{H}$-SiC wafers during chemo-mechanical polishing," Journal of Applied Physics, vol. 119, no. 13, Article ID 135702, 2016.

[4] H. N. Li, T. B. Yu, L. D. Zhu, and W. S. Wang, "Evaluation of grinding-induced subsurface damage in optical glass BK7," Journal of Materials Processing Technology, vol. 229, pp. 785-794, 2016.

[5] X. He, G. Wang, H. Zhao, and P. Ma, "Subsurface defect characterization and laser-induced damage performance of fused silica optics polished with colloidal silica and ceria," Chinese Physics B, vol. 25, no. 4, Article ID 048104, 2016.

[6] Z. Jia, Y. Su, B. Niu, B. Zhang, and F. Wang, "The interaction between the cutting force and induced sub-surface damage in machining of carbon fiber-reinforced plastics," Journal of Reinforced Plastics and Composites, vol. 35, no. 9, pp. 712-726, 2016.

[7] J. L. Rose, Ultrasonic Waves in Solid Media, Cambridge University Press, New York, NY, USA, 1999.

[8] D. Paehler, D. Schneider, and M. Herben, "Nondestructive characterization of sub-surface damage in rotational ground silicon wafers by laser acoustics," Microelectronic Engineering, vol. 84, no. 2, pp. 340-354, 2007.

[9] X. Cao, F. Jin, and I. Jeon, "Rayleigh surface wave in a piezoelectric wafer with subsurface damage," Applied Physics Letters, vol. 95, no. 26, Article ID 261906, 2009.

[10] C. H. Daros, "On modelling SH-waves in a class of inhomogeneous anisotropic media via the boundary element method," ZAMM-Zeitschrift für Angewandte Mathematik und Mechanik, vol. 90, no. 2, pp. 113-121, 2010.

[11] C. Potel, M. Bruneau, L. C. Foze N’Djomo, D. Leduc, M. Echcherif Elkettani, and J.-L. Izbicki, "Shear horizontal acoustic waves propagating along two isotropic solid plates bonded with a non-dissipative adhesive layer: effects of the rough interfaces," Journal of Applied Physics, vol. 118, no. 22, Article ID 224904, 2015.

[12] A. M. Gaur and D. S. Rana, "Dispersion relations for SH waves propagation in a porous piezoelectric (PZT-PVDF) composite structure," Acta Mechanica, vol. 226, no. 12, pp. 4017-4029, 2015.

[13] Y. Kong, J. Liu, and G. Nie, "Propagation characteristics of SH wave in an $\mathrm{mm} 2$ piezoelectric layer on an elastic substrate," AIP Advances, vol. 5, no. 9, Article ID 097135, 2015.

[14] Y. Pang, Y. Liu, J. Liu, and W. Feng, "Propagation of SH waves in an infinite/semi-infinite piezoelectric/piezomagnetic periodically layered structure," Ultrasonics, vol. 67, pp. 120-128, 2016.

[15] Y.-D. Li, T. Xiong, and Y. Guan, "Effects of coupled interfacial imperfections on $\mathrm{SH}$ wave propagation in a layered multiferroic cylinder," Ultrasonics, vol. 66, pp. 11-17, 2016.

[16] X. Y. Li, Z. K. Wang, and S. H. Huang, "Love waves in functionally graded piezoelectric materials," International Journal of Solids and Structures, vol. 41, no. 26, pp. 7309-7328, 2004.

[17] J. Liu, X. S. Cao, and Z. K. Wang, "Propagation of Love waves in a smart functionally graded piezoelectric composite structure," Smart Materials and Structures, vol. 16, no. 1, pp. 13-24, 2007.

[18] Z. Qian, F. Jin, Z. Wang, and K. Kishimoto, "Transverse surface waves on a piezoelectric material carrying a functionally graded layer of finite thickness," International Journal of Engineering Science, vol. 45, no. 2-8, pp. 455-466, 2007. 
[19] X. S. Cao, F. Jin, I. Jeon, and T. J. Lu, "Propagation of Love waves in a functionally graded piezoelectric material (FGPM) layered composite system," International Journal of Solids and Structures, vol. 46, no. 22-23, pp. 4123-4132, 2009.

[20] J. Zhu, W. Q. Chen, G. R. Ye, and J. Z. Fu, "Waves in fluid-filled functionally graded piezoelectric hollow cylinders: a restudy based on the reverberation-ray matrix formulation," Wave Motion, vol. 50, no. 3, pp. 415-427, 2013.

[21] J. G. Yu, B. Wu, and G. Q. Chen, "Wave characteristics in functionally graded piezoelectric hollow cylinders," Archive of Applied Mechanics, vol. 79, no. 9, pp. 807-824, 2009.

[22] Z. K. Wang and F. Jin, "Influence of curvature on the propagation properties of Rayleigh waves on curved surfaces of arbitrary form," Acta Mechanica Sinica, vol. 34, no. 6, pp. 895903, 2002.

[23] North Carolina State University, http://nanopatentsandinnova tions.blogspot.com/2010/02/smart-nano-coating-opens-doorto-safer.html. 

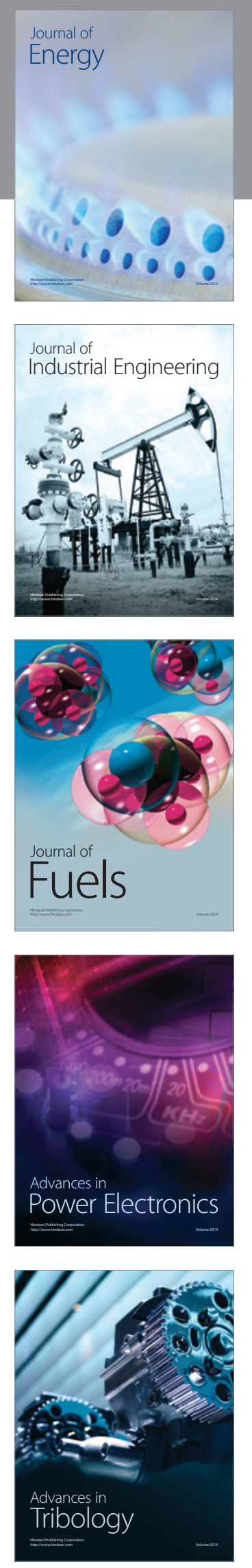
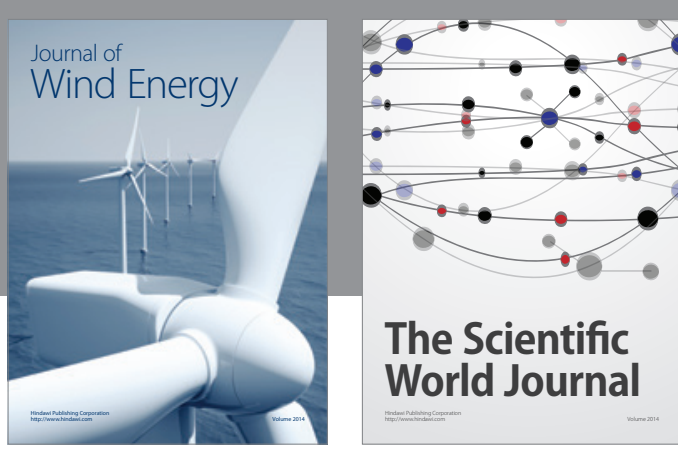

The Scientific World Journal
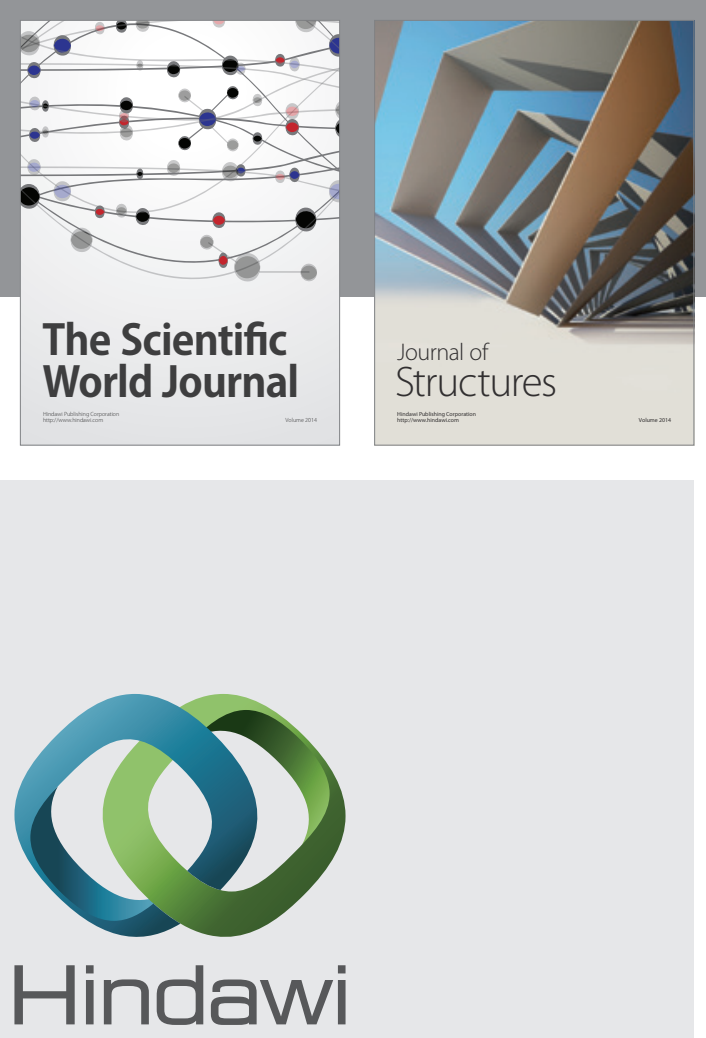

Submit your manuscripts at

http://www.hindawi.com
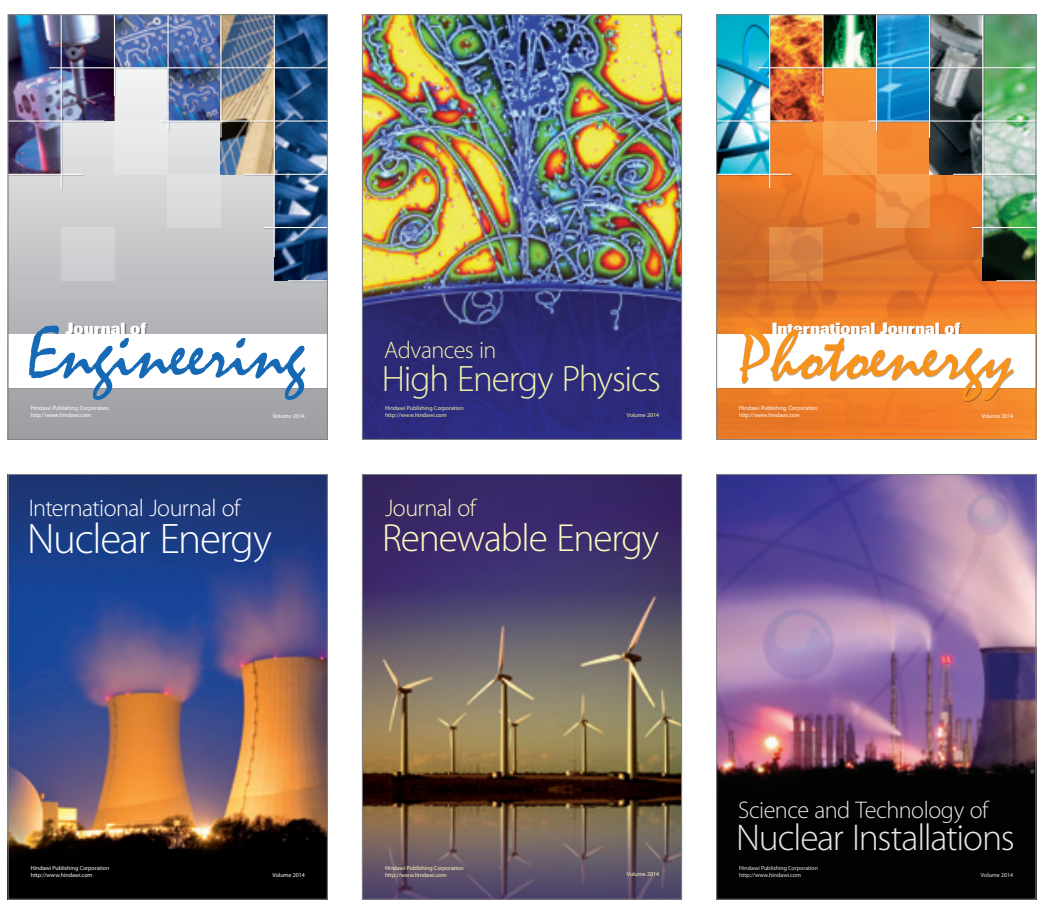
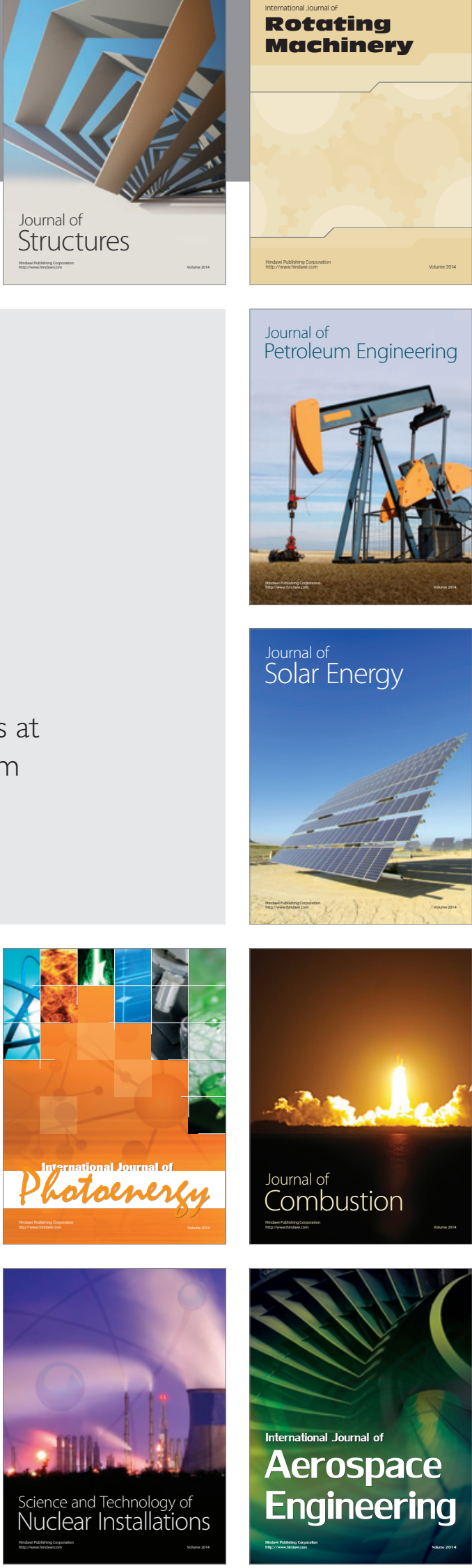